\title{
Neue Spieler in der Wissenschaft
}

\author{
Das Scheitern der traditionellen, disziplinär verengten Umweltforschung bei \\ der Lösung sehr komplexer Umweltprobleme förderte die Herausbildung eines \\ neven Forschungstyps: der Nachhaltigkeitsforschung, einer inter- oder trans- \\ disziplinären Wissenschaft, die im Spannungsfeld von Gesellschaft(-swissen- \\ schaften) und Natur(-wissenschaften) agiert. Bei ihrer Entstehung spielten \\ in Deutschland kleine, gemeinnützige Forschungsinstitute eine zentrale Rolle.
}

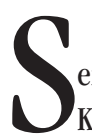
Von Thomas Jahn mehr und mehr als ein neues Leitbild globaler und regionaler Entwicklung. Im Nachhaltigkeitskonzept müssen ökonomische, soziale und ökologische Probleme im Zusammenhang gesehen und miteinander unter dem Postulat der Erhaltung von Entwicklungsmöglichkeiten für die $\mathrm{Zu}$ kunft verknüpft werden. Damit rückt die zukünftige Reproduktions- und Entwicklungsfähigkeit sowohl der Gesellschaft als auch ihrer natürlichen Lebensgrundlagen ins Zentrum des gesellschaftlichen Diskurses (1).

\section{- Normative Debatten}

Diese in der Folge auch in verschiedenen Ressorts der Politik geführten Debatten zur Nachhaltigen Entwicklung haben einen politisch-normativen Orientierungsrahmen entstehen lassen, der zahlreiche Forschungsaktivitäten ausgelöst hat sowohl in der Umweltforschung als auch in den ökologischen Sozialwissenschaften.

Inzwischen haben sich daraus neue Forschungsfelder wie ,angewandte Nachhaltigkeitsforschung“ und ,Sustainability Science“ entwickelt, denn wirtschaftliches oder gesellschaftliches Handeln, politische Eingriffe und technische Systemveränderungen unter dem Leitbild der Nachhaltigkeit können erst im Kontext und in Bezug auf wissenschaftliche Forschung und Reflexion entwickelt und bewertet werden. Nachhaltigkeitsforschung kann dabei zunächst als inter- oder transdisziplinäre Wissenschaft verstanden werden, die im Spannungsfeld von Gesellschaft(-swissenschaft) und Natur(-wissenschaft) agiert. Sie analysiert lebensweltliche Probleme unter der normativen Orientierung einer Nachhaltigen Entwicklung und erarbeitet spezifische Problemlösungen für unterschiedliche gesellschaftliche Gruppen.
Wo gesellschaftliche und ökologische Probleme im Orientierungsrahmen einer nachhaltigen Entwicklung zum Forschungsgegenstand gemacht werden, dort verändert sich das Forschungsfeld. So ist aus den umweltbezogenen Subdisziplinen der verschiedenen Sozialwissenschaften eine sozialwissenschaftliche Nachhaltigkeitsforschung am Entstehen. Sie entwickelt sich inzwischen zusammen mit der naturwissenschaftlich-technischen Umweltforschung zu einem neuen Forschungsgebiet, das sich immer mehr aus disziplinären Bindungen herauslöst.

\section{- Grenzen der Umweltforschung}

Die Umweltforschung war Anfang der 90er Jahre in eine Sackgasse geraten. Zum einen war die Diskrepanz zwischen den investierten Mitteln und den damit erzielten Erkenntnisgewinnen für gesellschaftliches Handeln unübersehbar geworden. Ein Beispiel dafür ist die Waldschadensforschung, die ständig neue Forschungsprobleme identifizierte und dafür immer mehr Mittel einforderte. Zum anderen war die Umweltforschung an ihre disziplinären Grenzen geraten, weil an die Stelle von der Untersuchung von Nachsorgetechniken wie Filtertechniken für Kraftwerke die Rufe nach präventiven Lösungskonzepten immer lauter wurden, die per se die Frage nach den gesellschaftlichen Akteuren stärker akzentuierten.

Im Nachhaltigkeitsdiskurs entstanden durch die Vermischung und Wechselwirkungen von den bisher eher getrennten Bereichen Ökologie, Ökonomie und Soziales neuartige, hybride und komplexe Forschungsprobleme. Demgegenüber verengt die traditionelle Umweltforschung die komplexen sozial-ökologischen Problemlagen in der Regel auf disziplinäre Probleme und kommt daher in ihrer naturwissenschaftlich geprägten Variante zu reduktionistischen verkürzten, in ihrer sozialwissenschaftlichen Variante zu sozial verkürzten Lösungs- vorschlägen. Sie verfehlt damit den komplexen Zusammenhang von gesellschaftlichen Handlungsmustern, natürlichen, technischen, ökonomischen und kulturellen Wirkungsgefügen in den gesellschaftlichen Naturverhältnissen.

\section{Ein never Forschungstyp}

In der offiziellen Karte der deutschen Forschungslandschaft finden sich bis Mitte der siebziger Jahre nur zwei große Sektoren: die klassische Hochschulforschung und die staatlich finanzierte außeruniversitäre Forschung. Doch die Karte ist nicht das Territorium. Seit der Gründung des Öko-Instituts in Freiburg vor 28 Jahren, als Ergebnis der Auseinandersetzungen um den Bau des Kernkraftwerkes Whyl, entstand - weitgehend unbeachtet durch die offiziellen Agenturen des Wissenschaftssystems - nach und nach eine neue Region in der Forschungslandschaft. Sie stellt gewissermaßen einen „Dritten Sektor“ kleiner, gemeinnütziger ökologischer Forschungsinstitute dar. Begonnen haben diese Institute als „Advocacy“-Wissenschaft - als eine Wissenschaft, die eng mit Bürgerinitiativen, den damals noch neuen sozialen Bewegungen und mit einzelnen Protestgruppen kooperierte. Inzwischen haben sich diese Institute stark verändert. Das Spektrum der Kooperationspartner ist breiter geworden und reicht bei einigen Instituten inzwischen bis zur Großindustrie. Teilweise sind die Institute näher an traditionelle Wissenschaftseinrichtungen und an einzelne akademische Disziplinen herangerückt, teilweise haben sie sich in Beratungseinrichtungen verwandelt.

In diesen Instituten hat sich eine neue Umweltforschung herausgebildet, die dem Nachhaltigkeitsziel verpflichtet ist. Sie öffnete sich einerseits zu Wirtschaft und Gesellschaft, andererseits richtete sie frühzeitig den analytischen Blick auf spezifische Problemausschnitte einer nachhaltigen Entwicklung, insbesondere auf verschiedene Aspekte eines nachhaltigen Wirtschaftens, nachhaltigen Konsums oder nachhaltiger Mobilität. In diesen Instituten hat sich der neue Forschungstyp der sozial-ökologischen Forschung und mit ihm ein neuer Modus der Wissensproduktion herausgebildet, der sich mit den Begriffen Problemorientierung, Akteursorientierung und Transdisziplinarität charakterisieren lässt.

Diese Forschung reagierte auf Defizite der vorwiegend disziplinär geprägten Umweltforschung und der sektoralen, stark interessensgebundenen Umweltpolitik mit einer interdisziplinären und integrativen Perspektive. In wissenschaftlicher 
Hinsicht hat sie sich darauf gerichtet, die noch immer weitgehend unverbundenen Erkenntnisse der naturwissenschaftlichen und der sozialwissenschaftlichen Umweltforschung sowohl problembezogen miteinander zu verknüpfen als auch theoretisch zu integrieren. Unter politischen und gesellschaftlichen Aspekten trägt sie der Tatsache Rechnung, dass Umweltpolitik immer stärker mit anderen Politikfeldern wie Wirtschafts-, Sozial-, Verkehrs-, oder Forschungs- und Technologiepolitik verflochten ist.

Damit bewegt sich diese Forschung an der Schnittstelle von Wissenschaft, Politik, Wirtschaft und Öffentlichkeit. Ihre allgemeinen Merkmale werden in der internationalen Diskussion unter dem Stichwort eines neuen Modus der Wissensproduktion, Mode 2, in zugespitzter Form zusammengefasst: Die gesellschaftliche Wissensproduktion findet immer stärker in unterschiedlichen Anwendungskontexten statt, ist transdisziplinär verfasst, erfolgt in vielfältig vernetzten und heterogenen organisatorischen Formen und in sozialer Verantwortung und bedarf von daher einer spezifischen Reflexivität (2).

Diese Ansätze einer Nachhaltigkeitsforschung spielten allerdings bislang im überwiegend disziplinär verfassten sowie auf sektorale und kurzfristige Politikvorgaben reagierenden Wissenschaftssysteme großenteils eine untergeordnete Rolle. Dazu hat die Tatsache beigetragen, dass förderpolitische Ansätze zu lange technikbezogen waren und weniger darauf zielten, das Potenzial dieses neuen Forschungstyps zu stärken und für die Entwicklung zukunftsfähiger gesellschaftlicher und politischer Handlungsmöglichkeiten zu nutzen.

\section{- Sozial-ökologische Forschung}

Aus der in den vorhergehenden Abschnitten kurz skizzierten Konstellation von gesellschaftlichem Problemdruck, Kritik an der etablierten Forschungslandschaft und Alternativen zur herrschenden Forschungspolitik ist so etwas wie ein Entwicklungsschub entstanden: In technik- und risikosoziologischen Diskursen, in der Umweltforschung selbst sowie in den gesellschaftlichen Debatten über Nachhaltige Entwicklung wurde ein forschungspolitisches Defizit sichtbar. Gleichzeitig wurde im Rahmen von MonitoringProzessen und durch die Evaluierung der Umweltforschung durch den Wissenschaftsrat aus dem etablierten Wissenschaftsbereich selbst die Kritik am Status Quo und die Forderung nach neuen Konzepten laut. Besonders deutlich artikulierte das ökoforum, ein Zusammenschluss von sieben deutschsprachigen Instituten aus dem dritten Forschungssektor, forschungspolitische Defizite und machte konkrete Veränderungsvorschläge (3). Es kam hier also gewissermaßen zu - durchaus auch nicht beabsichtigten - Resonanzen zwischen sehr unterschiedlichen Akteuren und Bereichen.

Nach dem Regierungswechsel Ende 1998 wurden mit der Entscheidung des BMBF, einen neuen Förderschwerpunkt für sozial-ökologische Forschung einzurichten, diese Defizite angegangen (4). Im Forschungsprogramm wurden drei vorrangige Förderziele formuliert und zu einem Gesamtkonzept verknüpft:

- die gezielte Förderung sozial-ökologischer Forschungsprojekte einschließlich einer kooperativen und kontrollierten Identifizierung des zukünftigem Forschungsbedarfs;

- die gezielte Förderung von kleinen, nicht-staatlichen und außeruniversitären Forschungseinrichtungen und die stärkere Vernetzung dieses dritten - Sektors des Wissenschaftssystems mit den Hochschulen und den staatlich finanzierten außeruniversitären Forschungseinrichtungen,

- die Initiierung und dauerhafte Etablierung eines für transdisziplinäre Forschung qualifizierten wissenschaftlichen Nachwuchses (5).

Inzwischen wurden mehrere Ausschreibungen in allen drei Förderbereichen durchgeführt; erste Ergebnisse liegen bereits vor (6).

Förderstrategisch sind für die sozial-ökologische Forschung drei Grundentscheidungen des Schwerpunktes entscheidend:

- Inhaltlich ist das neue Förderkonzept durch die Verknüpfung von konzeptioneller Klarheit und thematischer Offenheit geprägt. Diese Verknüpfung gelang im Rahmen der Konzeptentwicklung durch die Bestimmung einer wissenschaftlichen Problematik einer sozial-ökologischen Forschung, die vor allem durch eine Problem- und Akteursorientierung einerseits sowie eine integrative Perspektive andererseits gekennzeichnet ist. An diesen konzeptionellen Kern des Programms wurde ein breites Spektrum von inhaltlichen Fragen und Themen angelagert, durch die Forschende unterschiedlicher Sektoren, Disziplinen und Forschungsfelder angesprochen werden können.

- Bereits in der Phase der Konzeptentwicklung fand im Rahmen eines partizipativen und transparenten Prozesses eine enge Kooperation zwischen ausgewählten Expertinnen und Experten unterschiedlicher wissenschaftlicher Sektoren, dem BMBF und potenziellen Antragstellenden statt. Durch die Etablierung eines kooperativen Wis- sensnetzwerks und die Einrichtung von Diskursarenen konnte nicht nur auf einen breiten Wissensstand zurückgegriffen werden, sondern auch mögliche Interessendifferenzen und unterschiedliche Zielvorstellungen sichtbar gemacht und bearbeitet werden.

- Für den Förderschwerpunkt wurden ein Mix innovativer Förderbereiche definiert und neue geeignete Instrumente entwickelt. Neben der befristeten Förderung von Forschungsverbünden wurde auch eine Förderung strukturbildender Vorhaben ermöglicht, die an mittelfristige Ziele geknüpft sind und mit denen die Forschungskapazitäten unabhängiger und gemeinnütziger Forschungsinstitute gezielt gefördert werden. Außerdem wurde mit dem neuen Instrument der Sondierungsprojekte ein geeignetes Verfahren zur Themengenerierung entwickelt.

\section{Zwischenfazit}

Als (auch) staatlich geförderte Forschung verändert die sozial-ökologische Forschung das Verhältnis von Wissenschaft und Politik. Forschungsförderung bedeutet immer so etwas wie eine ,operative Kopplung" zwischen dem politisch-administrativen System und dem Wissenschaftssystem. Sie kann nur funktionieren, wenn die Förderprogramme Elemente enthalten, die in beiden Bereichen wirken und kommunikativ vermittelbar sind. Dies schließt aber aus, dass die geförderte Forschung einfach staatliche Auftragsforschung betreibt.

Mit dem neuen Förderschwerpunkt ist es in relativ kurzer Zeit mit relativ geringem materiellen und personellen Aufwand und in einem bis dahin einzigartigen Entwicklungsprozess gelungen,

- erstmals im Rahmen der existierenden BMBFFörderstruktur systematisch transdisziplinäre Forschung zu fördern,

- die erkennbare Benachteiligung einer bestimmten Gruppe von Forschungseinrichtungen offiziell anzuerkennen und deren Situation in einer mehr und mehr wettbewerblich verfassten Förder- und Auftragslandschaft in spürbarer Weise zu verbessern - wenngleich ,in the long run“ vermutlich noch nicht ausreichend, um die mit der Förderung verknüpften Erwartungen an sozial-ökologische Kompetenznetzwerke auch tatsächlich erreichen zu können,

- ein neues Verfahren der Entwicklung eines förderpolitischen Instruments beziehungsweise einer Maßnahme erfolgreich einzusetzen,

- die öffentlich geförderte Forschung - über sozial-ökologische Forschung hinaus - mit neuen inhaltlichen Herausforderung zu konfrontieren. 


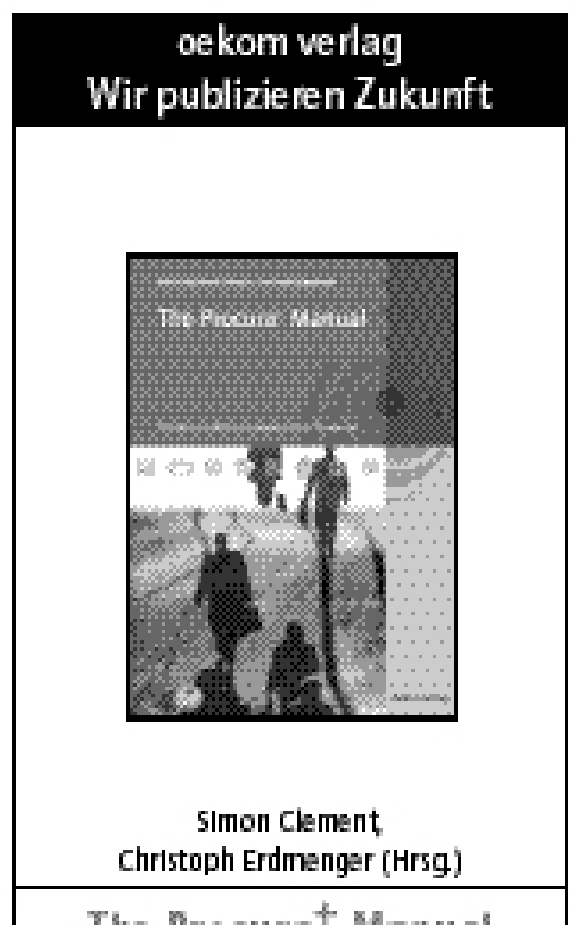

The Procura ${ }^{+}$Manual

A Gulde to costeffectwe Susta Inable Public Procurement

Sustainable public procuement is being inceasingly recognised across the world as a vital tool for ach ieving real ervironmental improvements and promoting patterns of sustainable productian and consumptian.

The Procura ${ }^{+}$Manua - $A$ Ginde to Cost-Effective Sustainolde Pullic Arocuvement has been written to provide European public procuse is with all they need to know to implement sustainable prouement in their authority - no matter what size or level of experience. It also ansuers several key questions including „Does it cost mare? $r$ " How do I decide what is a geen product?", and „Is it legal?, as well as providing key arguments for convincing othe rs with in an admini stration of its menits.

cekorm ve risg: Manchen 2004:

26B Seiten; 24,90 $\in$ (zod. Versundhosten im Auslard: 15BN 3936581-36-3

\section{Erhaitlich bai}

wni verlogsenire $\mathrm{CmbH} \& \mathrm{Ca} \mathrm{KG}$ Ausieler Ing celom verlag 85999 Lanthberg an lech Fon + 49/ (0) 8191/125.249, Fix -405 EMai f.cengivawniverlagsservice de
Das heißt, sich stärker der Erforschung komplexer, selbst organisierter, stark vernetzter und gekoppelter Systeme und Handlungszusammenhänge zuzuwenden und systematisch Werkzeuge der Integration zwischen den Disziplinen, zwischen Wissenschaft und Gesellschaft, aber auch zwischen verschiedenen Kulturen für den Gebrauch in Wissenschaft und Gesellschaft zu entwickeln.

\section{Ausblick}

Im Juni 2004 hat das Bundeskabinett mit dem Rahmenprogramm ,Forschung für die Nachhaltigkeit (FONA) “ eine Neuausrichtung der bisherigen Förderung der Umweltforschung des Bundes beschlossen. Die Forschungsförderung in diesem Bereich soll sich danach zukünftig am Leitbild einer Nachhaltigen Entwicklung orientieren und Beiträge zur Umsetzung der Nationalen Nachhaltigkeitsstrategie der Bundesregierung liefern. Die Forschungsthemen orientieren sich an gesellschaftlichen Problemen, die disziplinübergreifend und anwendungsorientiert bearbeitet werden sollen. Mit diesem Ansatz vollzieht das Rahmenprogramm die Entstehung der Nachhaltigkeitsforschung als neuem Forschungstyp nach und greift deren oben beschriebene Charakteristika im Kern auf. Bei den Förderinstrumenten, den begleitenden Strategien zu den Themen Bildung und Transfer wie auch bei der Ausgestaltung von FONA als „Lernendes Programm“ sind Rückgriffe auf die Konzepte und Erfahrungen im Rahmen des Förderschwerpunkts „,Sozial-ökologische Forschung“ erkennbar. Dies erlaubt eine positive Würdigung des neuen Rahmenprogramms als konzeptionellem Rahmen, um die erkennbaren Potenziale der Nachhaltigkeitsforschung für gesellschaftliche Problemlösungen zukünftig besser nutzbar zu machen und um dafür Instrumente der Forschungsförderung weiter zu entwickeln. Zwei kritische Punkte sind an dieser Stelle zu ergänzen: FONA bildet nur den Rahmen für die Projektförderung des BMBF im Bereich der Nachhaltigkeitsforschung. Im Bereich der institutionellen Förderung des Bundes dominieren dagegen noch immer naturwissenschaftliche, grundlagenorientierte Zugänge, zum Beispiel im Programm „Erde und Umwelt“ der Zentren der Helmholtz-Gemeinschaft. Die weitgehende Unverbundenheit beider Förderarten birgt die Gefahr eines Rückschritts, nämlich die der Desintegration von Naturwissenschaften und Sozialwissenschaften im Rahmen der geförderten Nachhaltigkeitsforschung. Hier stößt das Förderkonzept an institutionelle Begrenzungen der For- schungsorganisation und lässt kein Konzept erkennen, wie eine stärkere Integration der Forschung verschiedener Förderarten gelingen könnte. Weiter fehlen erkennbare Ansätze, wie die Ergebnisse und Methoden von FONA in anderen Förderschwerpunkten aufgenommen und umgesetzt werden könnten, etwa bei der Erforschung neuer Technologien, der Verkehrsforschung oder der IuK-Forschung - um nur einige Beispiele zu nennen. Eine konsequente Orientierung der Forschungsförderung am Leitbild einer Nachhaltigen Entwicklung auch in diesen Förderthemen wäre nach FONA der nächste Schritt.

\section{Anmerkungen}

(1) Dieser Artikel basiert in Teilen auf: Jahn, T.: Sozial-ökologische Forschung. Ein neuer Forschungstyp in der Nachhaltigkeitsforschung. In: Linne, G./ Schwarz, M. (Hg.), Handbuch Nachhaltige Entwicklung. Wie ist nachhaltiges Wirtschaften machbar? Opladen: Leske + Budrich 2003, S. 545-555.

(2) Gibbons, M. et Al.: The New Production of Knowledge. The dynamics of science and research in contemporary societies. London 1994.

(3) Ökoforum (Hg.): Technische Scheuklappen. Memorandum des Ökoforums zur Umweltforschung. In: Politische Ökologie Nr. 52, 1997, S. 90-91. Ökoforum (Hg.): Critical Analysis of the Sixth Framework Programme for Research and Development from the Perspective of Transdisciplinary Research. Frankfurt am Main/Freiburg 2001.

(4) Becker, E./Jahn, Th./Schramm, E.: Soziallökologische Forschung. Rahmenkonzept für einen neuen Förderschwerpunkt. Gutachten im Auftrag des BMBF. Studientexte des Instituts für sozial-ökologische Forschung Nr.6. ISOE, Frankfurt am Main 2000.

(5) BMBF (Hg.): Rahmenkonzept Sozial-ökologische Forschung. Bonn 2000.

(6) Balzer, I./ Wächter, M.: Sozial-ökologische Forschung. Ergebnisse der Sondierungsprojekte aus dem BMBF-Förderschwerpunkt. München 2002.

\section{Der Autor}

Dr. Thomas Jahn ist Leiter des Instituts für sozialökologische Forschung (ISOE).

Kontakt: ISOE, Hamburger Allee 45, 60486 Frankfurt, Tel.: 069/70769190, E-Mail: jahn@isoe.de, Internet: www.isoe.de 
(c) 20I0 Authors; licensee IÖW and oekom verlag. This is an article distributed under the terms of the Creative Commons Attribution Non-Commercial No Derivates License (http://creativecommons.org/licenses/by-nc-nd/3.o/), which permits unrestricted use, distribution, and reproduction in any medium, provided the original work is properly cited. 